\title{
Planet Detection using the Advanced Camera for Surveys on the Hubble Space Telescope
}

\author{
William B. Sparks, John Krist \& Mark Clampin \\ Space Telescope Science Institute, 3700 San Martin Drive, Baltimore, \\ MD 21218, U.S.A. \\ Holland Ford \\ Johns Hopkins University, 3400 N. Charles Street, Baltimore, \\ MD 21218, U.S.A.
}

\begin{abstract}
The Advanced Camera for Surveys was installed on the Hubble Space Telescope in March 2002. The instrument science team will search for planets using direct imaging with the ACS coronagraph and with precise astrometric and photometric measurement of suitable stars and disks. The camera is operational and performing to expectation.
\end{abstract}

\section{The Advanced Camera for Surveys}

The Advanced Camera for Surveys (ACS) is a new instrument on the Hubble Space Telescope (HST) built under the guidance of a science team led by $\mathrm{H}$. Ford (JHU). The goal is to provide a diverse suite of imaging capabilities with performance that represents an increase of over a factor ten for HST (Ford et al. 1996). The ACS is three cameras with some shared optics. There are two optical imagers, a wide field camera (WFC) and a high resolution camera (HRC). The WFC is a high throughput, sensitive, $I$-band optimized camera designed for faint survey observations. The camera employs two SITe CCDS each $2048 \times 4096$ pixels butted together to give a $4096 \times 4096$ pixel field of view, $\approx 200 \times 200$ arcsec areal coverage. The HRC is optimized for blue imaging, critically sampled across much of the optical wavelength region, utilizing a single $1024 \times 1024$ SITe CCD. The camera offers a field of view $\approx 26 \times 29$ arcsec on the sky, and has been specially coated to provide good sensitivity into the near-UV $(\sim 200 \mathrm{~nm})$. In addition to the two "optical" cameras, there is a third "solar blind camera" (SBC) which is optimized to provide good sensitivity in the far-UV, from 115 to $170 \mathrm{~nm}$, which will not be considered further here.

A series of spectacular images were obtained early in the ACS mission, and are publically available through the STScI web site (www.stsci.edu), see e.g. Fig. 1. These include dramatic images of merging galaxies and nebulae. While the field of view is wide, the spatial resolution remains very high, and the improved sensitivity already results in images not unlike the famous "Hubble Deep Field" (Williams et al 1996). It is interesting to consider that galaxies like these may be quite representative of the conditions under which the bulk of star and presumably planet formation took place. 


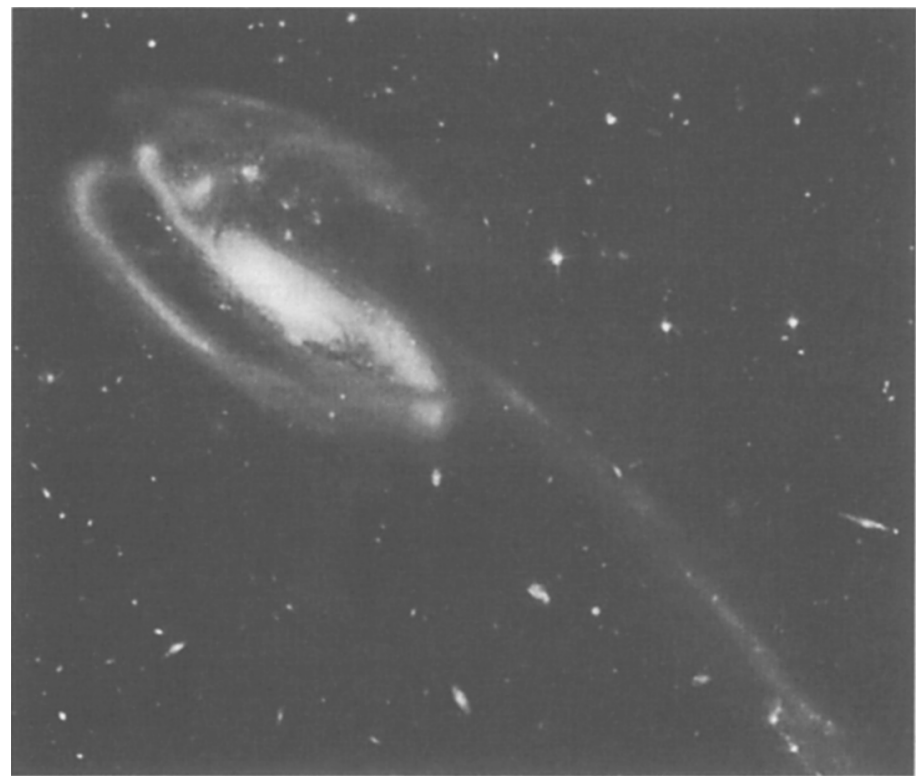

Figure 1. Early release image of interacting galaxy taken with ACS Wide Field Camera.

\section{The ACS Aberrated Beam Coronagraph}

There are a variety of special modes and configurations available with ACS, including grism and prism spectroscopy, narrow-band imaging using "linear ramp filters" across the entire optical wavelength range, polarization and coronagraphy. For extrasolar planet imaging, it is the coronagraphic capability that is of the greatest interest. An overview of the ACS optics, including the observed performance of the coronagraphic mode is available from STScI in the "Advanced Camera for Surveys Instrument Handbook". The "aberrated beam coronagraph" is a moveable mechanism attached to the calibration assembly at the entrance aperture of the ACS. When inserted it places two occulting masks in the HST focal plane, while apodizing the beam at the nearby pupil. There is also an occulting finger close to the CCD focal plane intended to eliminate charge bleeding from the bright central spot which arises because the occulting spots are in the aberrated beam. Fig. 2 shows a preliminary measurement of the performance of the coronagraph. The wing suppression is quite effective but limited by an inability to correct for mid-range wavefront errors known to exist on the HST mirrors. Additional point spread function (PSF) wing suppression is obtained using "roll-deconvolution". Using this technique, images are obtained with the telescope at two different roll angles. All the telescope and instrument optics remain fixed and the PSF on the detector remains fixed, however any underlying astronomical target moves with respect to the detector. Hence a simple subtraction, in principle, is an exceedingly powerful way to eliminate many of the complexities of the HST PSF.

The ACS Instrument Handbook provides more information and images relating to the characteristics and use of the coronagraph. 


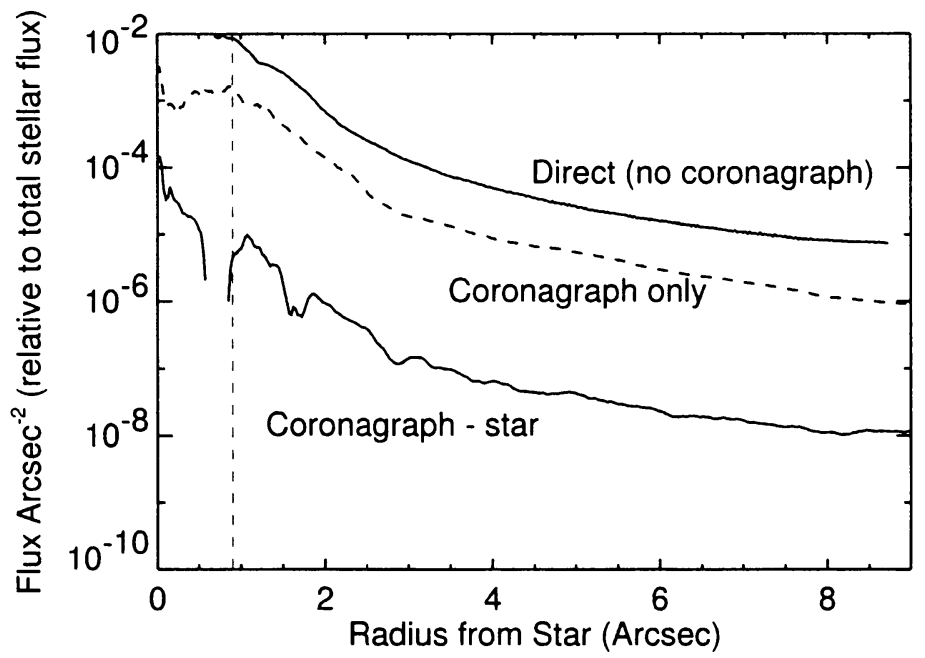

Figure 2. Preliminary measurement of the performance of the ACS coronagraph small spot at $435 \mathrm{~nm}$. Please refer to the ACS Instrument Handbook for up-to-date information.

\section{Scientific Program}

\section{1. $\alpha$ Centauri}

The nearest stellar system to the Sun is that of $\alpha$ Centauri, a triple system comprising $\alpha$ Centauri A and B and Proxima Centauri at significantly greater separation. Since it is the nearest star, and both A and B components are like the Sun, it is an obvious target for extrasolar planet searches. Hypothetical planets of given properties (contrast ratio, typically $\sim 10^{-9}$, distance from star) will be at wider separation and be brighter in a nearer star, and hence easier to detect. Components A and B are separated by $23.4 \mathrm{AU}$ (semimajor axis) with luminosities 1.6 and $0.45 L_{\odot}$ respectively.

The fact that $\alpha$ Centauri is a multiple system is a possible complication since this may reduce or remove the dynamical stability of planetary orbits. Benest (1988) and Wiegert \& Holman (1997) concluded that stable orbits can exist within the system.

The observing strategy will either be roll-deconvolution, described above, or will make use of the wavelength dependence of the PSF using "spectral deconvolution" (Sparks \& Ford 2002). Sparks et al. (2000) show a simulated set of images of Jupiter placed at the distance of $\alpha$ Centauri which demonstrate that under very favourable circumstances, it should just be feasible to image that planet using the ACS coronagraph.

\subsection{G1 229B}

Gl $229 \mathrm{~B}$ is a brown dwarf, mass only $\approx 45 M_{J}$ ("J" denoting Jupiter) orbiting Gl $229 \mathrm{~A}$, and size $R \sim 1 R_{J}$. These two properties offer high leverage on traditional techniques. Firstly, the low mass of the brown dwarf means that there would be a relatively large astrometric reflex motion induced by the orbital mo- 
tion of a planetary companion to the brown dwarf. Such motion is potentially detectable. The ACS offers high spatial resolution and sensitivity to greatly improve the effectiveness of observations of this type. Secondly, the small size of the brown dwarf means that transits by smaller planetary bodies would give a strong photometric signal. For example, an Earth sized planet at the Roche limit would give a potentially measurable $1 \%$ photometric drop in flux from the brown dwarf during a transit. The high ACS sensitivity means that short integration times are needed and hence good time resolution may be obtained for studies of this type.

\subsection{Dust Disks}

The coronagraphic and imaging modes of the HRC will be used to study circumstellar disks. Disk images may be used to characterize disk morphology over all scales, including warps and regions of enhanced or depleted density and to betray the presence of imbedded planets through fine-scale structures, gaps and warps. Mouillet et al. (2001) used similar (HST/STIS) observations of the disk around HD 141569 to reveal a very structured, asymmetrical system with arcs and gaps. It is possible that the disk properties are due to the gravitational perturbation of a companion.

\section{Summary}

The Advanced Camera for Surveys has been installed in the Hubble Space Telescope and is performing to expectation. Three scientific projects will be undertaken by the ACS science team that address extrasolar planets. The first will be attempted direct imaging detection of Jupiter-sized planets around $\alpha$ Centauri. The second is a series of observations of the brown dwarf Gl 229B to seek astrometric reflex motion and photometric occultation events that could probe much smaller planet masses. Thirdly, imaging of dust disks around stars will be used to seek evidence of ripples, rings and gaps indicative of planets within the system.

\section{References}

Benest, D. 1988, A\&A, 206, 143

Ford, H.C., et al. 1996, SPIE, 2807, 184

Mouillet, D., Lagrange, A. M., Augereau, J. C., \& Ménard, F. 2001, A\&A, 372, L61

Sparks, W. B., Brown, R., Burrows, C., Clampin, M., \& Krist, J. E., 2000, in ASP Conf. Ser. 213, Bioastronomy '99: A New Era in the Search for Life ed. G. Lemarchand \& K. Meech, 131

Sparks, W. B., \& Ford, H. C. 2002, ApJ, 578, 543

Wiegert, P. A., \& Holman, M. J. 1997, AJ, 113, 1445

Williams, R. E., et al. 1996, AJ, 112, 1335 